\title{
The control of maize spikelet meristem fate by the APET ALA 2-like gene indeterminate spikelet1
}

\author{
George Chuck, ${ }^{1}$ Robert B. Meeley, ${ }^{2}$ and Sarah Hake ${ }^{1,3,4}$ \\ ${ }^{1}$ Department of Plant and Microbial Biology, University of California, Berkeley, California 94720, USA; ${ }^{2}$ Pioneer Hi-Bred \\ International, Johnston, Iowa 50131, USA; ${ }^{3}$ Plant Gene Expression Center, US Department of Agriculture-Agricultural \\ Research Service (USDA-ARS), Albany, California 94710, USA
}

The orderly production of meristems with specific fates is crucial for the proper elaboration of plant architecture. The maize inflorescence meristem branches several times to produce lateral meristems with determinate fates. The first meristem formed, the spikelet pair meristem, produces two spikelet meristems, each of which produces two floral meristems. We have identified a gene called indeterminate spikelet1 (ids1) that specifies a determinate spikelet meristem fate and thereby limits the number of floral meristems produced. In the absence of ids1 gene function, the spikelet meristem becomes indeterminate and produces additional florets. Members of the grass family vary in the number of florets within their spikelets, suggesting that ids1 may play a role in inflorescence architecture in other grass species. ids1 is a member of the A PETALA 2 (AP2) gene family of transcription factors that has been implicated in a wide range of plant development roles. Expression of ids1 was detected in many types of lateral organ primordia as well as spikelet meristems. Our analysis of the ids1 mutant phenotype and expression pattem indicates that ids1 specifies determinate fates by suppressing indeterminate growth within the spi kelet meristem.

[Key Words: Meristem; spikelet; indeterminate spikelet1; APETALA2; determinacy]

Received N ovember 3, 1997; revised version accepted February 26, 1998.

The devel opment of the plant body is dependent on the activity of apical meristems, groups of indeterminate cells found at the growing tips. The ability of shoot apical meristems to remain indeterminate allows them to continually initiate the organs, tissues, and secondary meristems required for normal development. This property requires that the meristem set aside a population of cells to renew itself and replenish the cells lost to each lateral organ or secondary meristem. The failure of this process to occur results in the termination of the growing tip. Some meristems, such as floral meristems, are consumed in the production of lateral organs, and can be described as having a determi nate fate. The infl orescence of maize is particularly useful for studying meristem determinacy as it undergoes several well defined branching events to produce meristems with increasingly restricted fates.

Grass florets are organized into units called spi kelets, each consisting of a pair of sterile bracts called glumes enclosing a fixed number of florets (Clifford 1987). The regulation of floret number per spikel et is a prime determinant of spikelet architecture among members of the grass family. The spikel et of maize is determinate, pro-

${ }^{4}$ Corresponding author.

E-MAIL maizesh@nature.berkeley.edu; FAX (510) 559-5678. ducing only two florets in defined positions on an axis called the rachilla (Weatherwax 1923). Relatives of maize are characterized by spikelets with an indeterminate number of florets, such as wheat, or contain only one floret per spikelet, as in barley. One classical criterion used to di stinguish different grass species is whether their spikelets contain a determinate or indeterminate number of florets (Clifford and Watson 1977). The spikelet itself is but one component of the complex branched inflorescence of maize. In contrast to dicotyledonous plants such as Arabidopsis in which flowers are produced directly by the apical and lateral meristems (Hempel and Feldman 1994), there are at least two distinct inflorescence branching steps in maize before the spikel et meristem terminates in the production of two florets. These extra branching steps allow for greater morphological diversity among the grasses.

A number of mutants have been described in maize that result in an extra number of florets within the spikelet (Veit et al. 1993). In the branched silkless mutants, extra florets are initiated in male tassel spikel ets (Kempton 1934), although a more extreme transformation is seen in female spikelets of the ear in which florets are transformed into long indeterminate branches (Veit et al. 1993). Studies of the Tasselseed6 mutation reveal ed that the spikel et meristem undergoes a delay in acquiring de- 
terminacy, allowing it to initiate florets for a longer period of time (Irish et al. 1994). The analysis of Tasselseed6 mutants led to a model (Irish 1997) in which the inflorescence meristem and its branch derivatives pass through an orderly, defined series of determinate developmental states, ending with the conversion of the terminal spikelet meristem into the upper floret. Similar branching mutants have also been described in other grass species and include the multiflorous mutation of barley (Bossinger et al. 1992) and the dominant Naked mutant of oats (Ougham et al. 1996).

An abundance of genetic and molecular studies have identified several genes important for floral development. One such gene, the APETALA2 (AP2) homeotic gene of Arabidopsis, has several functions in flower, seed, and ovule devel opment (Kunst et al . 1989; J ofuku et al. 1994; M odrusan et al. 1994). In addition to its role in determining floral organ identity, AP2 affects the regulation of floral meristem identity. For example, double mutants of the weak ap2-1 allele with floral meristem identity mutants such as leafy or apetalal produce more cofl orescence si de branches in the place of flowers (Bowman et al. 1993). Also, weak ap2 alleles under short days cause the formation of tertiary floral shoots in the axils of transformed sepals (Schultz and Haughn 1993). The AP2 gene belongs to a large gene family, 12 of which have been identified in Arabidopsis (Okamuro et al. 1997). N umerous homol ogs have been identified in both monocots and dicots (Jofuku et al. 1994; Ohme-Takagi and Shinshi 1995; M oose and Sisco 1997). Mutations in the AP2-like gene, AINTEGU MENTA, are defective in ovule development (Elliot et al. 1996; Klucher et al. 1996). The glossy15 gene of maize has recently been shown to be an AP2-like gene that functions to repress adult leaf characters in juvenile leaves (M oose and Sisco 1997).

Here we describe a new branching mutant in maize, indeterminate spikel etl (ids1), that we obtained by analyzing the phenotype conditioned by loss of function of a maize AP2-like gene. ids1 mutants have an indeterminate spikelet in which several florets are produced instead of the two found in wild-type maize. idsl expression was observed in a variety of lateral organs as well as the spikelet pair and spikel et meristems. Our analysis indicates that the idsl gene is critical for the regulation of spikel et meristem determinacy in maize.

\section{Results}

Isolation of the idsl gene

The AP2 gene from Arabidopsis was used to screen two maize cDNA libraries at low stringency, one prepared from immature ears and the other from vegetative meristems. The same class of cDN A was isolated from each library. The longest clone of this class was 1967 nucleotides and contained an ORF of 433 amino acids (Fig. 1) with several domains showing striking amino acid similarity to the AP2 gene of A rabidopsis (J ofuku et al. 1994). T wo tandemly repeated 68 amino aci d motifs were found

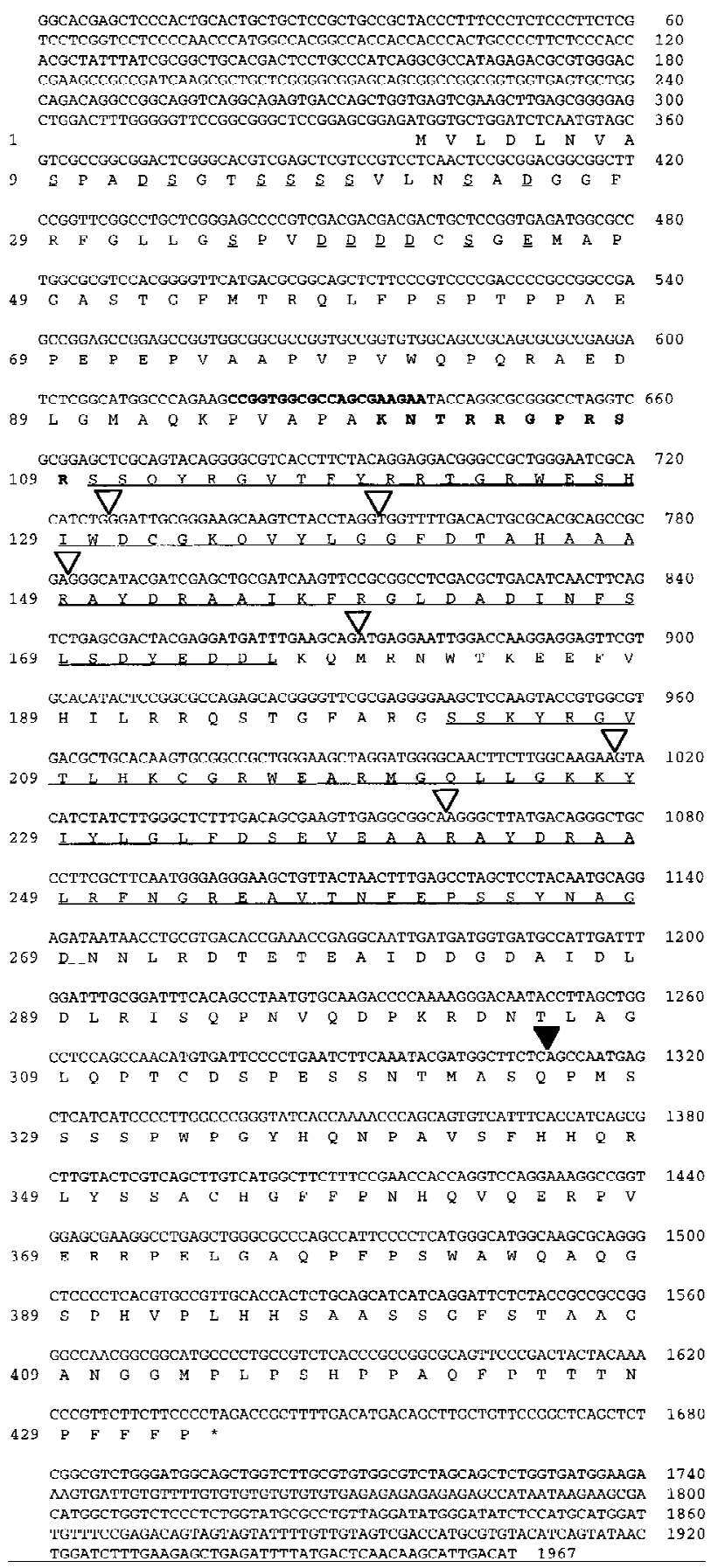

Figure 1. Nucleotide and deduced amino acid sequence of IDS1. Amino acid numbers are shown to the left; nucleotides are numbered on the right. Underlined amino acid sequences represent the two AP2 domains (Jofuku et al. 1994). Serines and acidic amino acids at the amino terminus are indicated with short underlines. Amino acid sequences in bold represent the basic region with putative nuclear localization activity. Bold nucleotide sequences correspond to the ZAP2-1 primer used for the PCR screen. $(\nabla)$ The six intron positions conserved between IDS, AP2, and GL15; $(\boldsymbol{\nabla})$ the other intron.

that share $86 \%$ amino acid identity with the AP2 domain of the A rabidopsis AP2 protein. TheAP2 gene fam- 
ily can be classified into two groups, designated as EREBP-like or AP2-like, on the basis of whether they possess one or two of the AP2 repeats, respectively (Okamuro et al. 1997). The IDS1 protein belongs to the latter class that includes the AP2, AINTEGUMENTA, GLOSSY 15, and RAP2.7 proteins (Fig. 2) Jofuku et al. 1994; Klucher et al. 1996; Moose and Sisco 1997; Okamuro et al. 1997). The tobacco ERE-BP proteins, which have only one of these repeats, bind DN A (Ohme-Takagi and Shinshi 1995). Thus, by anal ogy it is likely that IDS1 functions as a transcription factor. In support of this, a short stretch of basic amino acids that may function as a nuclear local ization domain is present in the IDS protein between animo acids 100 and 110 (Fig. 1). The AP2 protein from Arabidopsis contains a serine-rich acidic domain at the amino terminus that may function as an activation domain (Jofuku et al. 1994). Although a simiIar region is found at the amino terminus of the IDS1 protein at positions 9-45, the region is much smaller and has fewer acidic and serine residues compared with AP2. Outside the AP2 domain there is very little sequence similarity between AP2 and IDS1. Given this finding, it is surprising that the positions of six introns within the AP2 domain are conserved between ids1 and AP2 (Fig. 1). A similar result was found for the $\mathrm{gl} 15$ gene of maize (Moose and Sisco 1997).

ids1 was mapped to the long arm of chromosome one at position 192 by use of recombinant inbred lines (Burr et al. 1988) probed with a nonrepetitive DN A fragment from the $3^{\prime}$ end of idsl. No known morphological mutants have been mapped to this position.

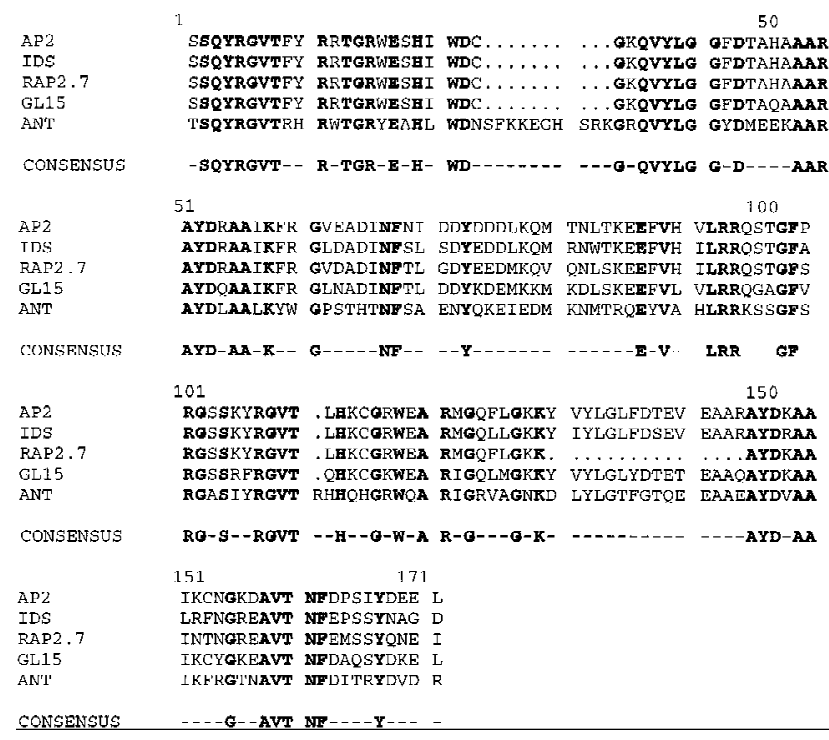

Figure 2. Comparison between the AP2 domains of the AP2like genes. The deduced amino acid sequences of the AP2 domains of IDS1 (GenBank accession no. AF048900), RAP2.7 (AF003100), GL15 (U 41466), and ANT (U 40256) are compared with AP2 (ATU12546). The region in between the two AP2 repeats, i.e., the linker region, starts at amino acid 78 and ends at 102. Gaps, represented by dots, were introduced to facilitate the alignment. Amino acids shared by all five proteins are shown in boldface type and on the consensus sequence at bottom.
Isolation of recessive idsl mutations

To elucidate the function of ids1, we used a reverse genetic strategy to generate loss-of-function alleles. PCR was used on a large population of plants carrying Mutator $(\mathrm{Mu})$ transposons to find insertions into ids1 (Bensen et al. 1995; Meeley and Briggs 1995). Primers flanking the AP2 domain of ids1 in combination with Mu primers yielded two independent insertion events through this screen. Sequencing of the PCR products localized the insertions more precisely. As shown in Figure 3, the Mu insertion in ids1-muml was localized to the fifth conserved intron of the AP2 domain, whereas the ids1mum2 insertion was localized to the first conserved intron 6 bp away from a splice acceptor site. Because Mu elements generate a 9-bp duplication upon insertion (Bennetzen et al. 1993), the splice acceptor site is also found at the $5^{\prime}$ end of $\mathrm{Mu}$.

Lines carrying the ids1-mum1 and ids1-mum2 alleles displayed a recessive floral phenotype that was more severe in ids1-mum2 lines. To verify that the insertions into ids1 were responsible for the floral phenotype, we anal yzed DN A from 104 F2 plants using the ids1 gene as a probe on DNA gel blots. Cosegregation of novel RFLPs with the floral phenotype was observed for 29 chromosomes containing the ids1-mum1 all ele and 91 chromosomes containing ids1-mum2 (data not shown). No recombinants between the floral phenotype and ids1-mumspecific polymorphisms were found for either allele.

We analyzed the phenotypes of ids1-mum plants following a backcross into A632 and self-pollination. The most consistent phenotype in ids1-mum mutants was a defect in the spikel et. Mature wild-type tassel spikel ets contain two florets each consisting of three stamens and two lodicules enclosed within palea and lemma bracts (Fig. 4B). The palea is distinguishable from the lemma by virtue of its position and the fact that it is bi-keel ed with a double midrib (Clifford and Watson 1977). The spikelets of the ids1-mum2 tassels are larger than those of wild type (Fig. 4A) because of the fact that extra florets are present (Fig. 4C). The number of florets ranges from at least 3 in ids1-muml plants to as many as 10 in ids1mum2 plants. In addition to these florets, a thin rachilla containing several smaller florets can usually be seen in between the two uppermost florets (Fig. 4F). These smaller florets tend to become progressively reduced, such that the ones remaining at the tip of the rachilla are barely visible. The larger extra florets show the normal pattern of sexual differentiation and have the correct number of stamens and lodicules surrounded by palea and lemma (Fig. 4C).

The ids1-mum female spikelets also exhibit a larger size as a result of the presence of extra florets (Fig. 4D,E). $\mathrm{N}$ ormal female spikelets contain only a single mature floret because of the abortion of the lower floret (Delong et al. 1993). The development of secondary sexual characteristics in the female florets include the abortion of stamens and the development of the gynoecium, as exempl ified by the formation of long silks composed of two fused carpels (Randol ph 1936). ids1-mum1 female spike- 
Figure 3. Localization of the Mu elements in ids1-mum1 and ids1-mum2. ids1 intron and exon sequences are shown in normal and boldface lettering, respectively. Intron and exon numbers are shown above the DN A sequences. The underlined sequences represent the characteristic 9-bp duplication associated with $\mathrm{Mu}$ insertions.

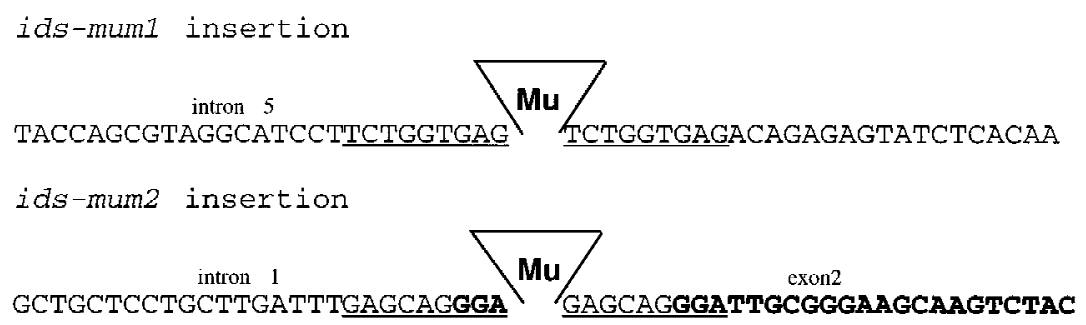

lets often contain more than one silk located at ectopic positions (Fig. 4D). These silks, however, usually do not develop normally; they are often unfused and fail to elongate to their proper length. If the developing ear is manually opened and pollinated, a few kernels develop, suggesting that all other aspects of fertilization and fruit development are normal. When grown to maturity, these kernels display idsl-mum homozygous phenotypes, and so do not represent germinal excisions of the $\mathrm{Mu}$ elements. The ids1-mum2 allele appears more severe, in that most of the extra florets fail to devel op fully formed lateral organs (Fig. 4E, left). Approximately $5 \%$ of the ids1-mum2 female spi kel ets show an el ongating rachilla that emerges from the spikel et (Fig. 4F). In such cases, it can be observed that the rachilla does not terminate in the uppermost floret further confirming its indeterminate nature. $\mathrm{N}$ o obvious and consistent phenotypes were seen in the leaves and roots of idsl mutants despite the fact that idsl is expressed in those organs.

\section{Scanning electron microscopy analysis}

Scanning electron mi croscopy was used to determine the point at which ids1-mum spikelet meristem development diverges from that of wild type. The inflorescence meristem normally initiates spikelet pair meristems acropetally, which, in turn, branch to form two spikelet meristems (Fig. 5A) (Cheng et al . 1983). The spikelet me- ristems also branch to form the lower and upper florets. These florets initiate in a distichous pattern, that is, alternating 180 degrees from one another (Fig. 5B). Although the lower floral meristem initiates first, lateral organ devel opment occurs in the upper floret much earlier than in the lower floret (Fig. 5C,D). The pal ea is the first lateral organ to differentiate, and the three stamens develop soon after (Cheng et al. 1983). Sexual differentiation occurs at later stages in which the gynoecium aborts in male florets and the stamens abort in female florets (Dellaporta and Calderon-U rrea 1993).

idsl-mum mutants show normal development until the stage at which the spikelet meristem begins to branch. At this point, the idsl-mum spikelet meristems appear slightly el ongated (Fig. 5E, top). In slightly older spikelet meristems (Fig. 5E, middle), two distinct branching events can be seen as shown by the presence of two lemmas (arrows). Floral meristems then form in the axils of these lemmas (Fig. 5E, bottom, and F). These branching events occur on opposite sides of the spikelet meristem in a distichous pattern. The spi kel et meristem persists after each branching event and remains in between the two last formed florets (Fig. 5G,H). This residual spikelet meristem continues to initiate extra lemmas and florets distichously (Fig. 5F,G). The rate of lateral organ development between the first two ids1-mum florets is similar (Fig. $5 \mathrm{H}$ ), in contrast to wild type in which the organs in the upper floret devel op first.
Figure 4. Phenotypes in idsl-mum male and female spikelets. (A) N ormal A632 tassel spikelet (left) and ids1-mum2 tassel spikelet (right). (B) Dissected normal A632 tassel spikelet showing lateral organs of upper and lower florets. (C) Dissected ids1-mum2 tassel spikelet showing four florets instead of two. Each floret has three stamens surrounded by palea and lemma as in A632. (D) N ormal A632 ear spikel et (left) and ids-muml ear spikelet (right). Arrowheads point to silks. (E) Median Iongitudinal sections through unfertilized 25-day-old ids1-mum2 ear spikelet (left) and A632 ear spikelet (right). Black arrows point to florets. A632 spikelets have a single floret with an enlarged nucellus; ids1-mum2 ear spikelets have at least three florets with greatly reduced lateral organ development. (F) ids1-mum2 ear spikelet with an elongating rachilla containing two florets. The single arrowhead points to the tip of the rachilla. (st) Stamen; (il) inner lemma; (pa) pal ea; (ol) outer lemma; (ig) inner glume; (og) outer glume; (uf) upper floret; (If) lower floret; (ra) rachilla; (fl) floret.
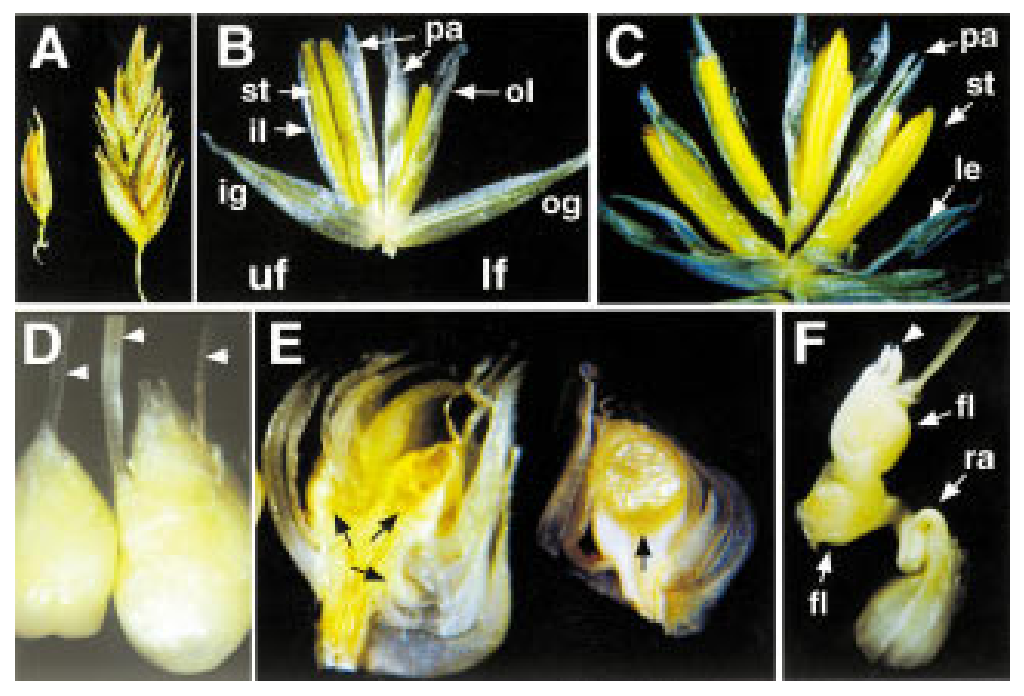

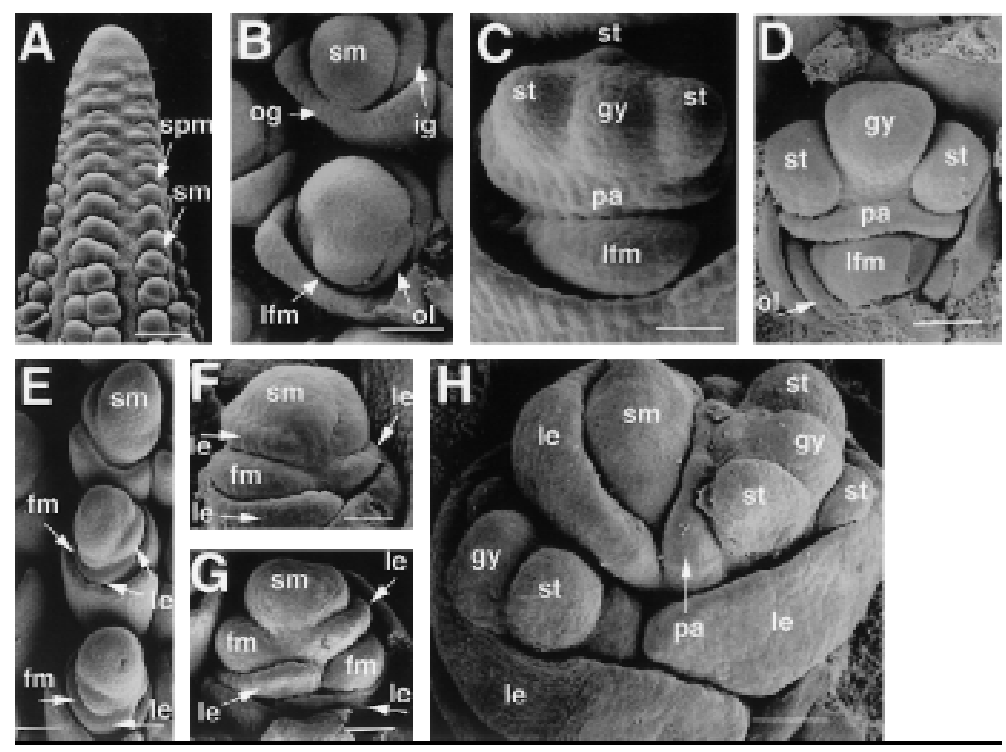

Figure 5. Scanning electron microscopy of normal and ids1-mum2 ears. (A) A632 ear primordium with initiating spikelet pair primordia and spikelet primordia. Bar, $300 \mu \mathrm{m}$. (B) A632 unbranched spikelet meristem (top) and slightly older branched spikelet meristem (bottom). The older spikel et meristem undergoes lateral branching to initiate the lower floret. Bar, $102 \mu \mathrm{m}$. (C) Development of lateral organs in A632 ear spikelets. The lateral organs of the upper floret devel op first. Bar, $80 \mu \mathrm{m}$. (D) M aturing lateral organs of the upper floret of A632 ear spikelets with initiating gynoecial ridge. Bar, $104 \mu \mathrm{m}$. (E) Branching ids1-mum2 spikelet meristems. The uppermost spikelet meristem has not branched yet and appears el ongated. Si multaneous floret branching and lemma initiation occurs on opposite sides of the middle and lower spikelet meristems. Bar, $156 \mu \mathrm{m}$. (F) Further devel opment of ids1-mum2 spikel et meristems with glumes removed showing development of the floral meristems within the axils of the lemmas. The spikelet meristem persists after each branching event. Bar, $58 \mu \mathrm{m}$. (G) Older ids1-mum2 spikel et meristem with gl umes removed. The resi dual spikel et meristem el ongates to initiate another floret. Bar, $60 \mu \mathrm{m}$. (H) ids1-mum2 spikel et showing maturing lateral organs. There is no distinction between upper and lower floret in terms of lateral organ devel opment. The youngest floret branching from the spikelet meristem is obscured by the lemma. Bar, $171 \mu \mathrm{m}$. (spm) Spikelet pair meristem; (sm) spikel et meristem; (Ifm) lower floral meristem; (gy) gynoecium; (fm) floral meristem; (le) lemma.

We used the knotted1 (knl) homeobox gene of maize, which is expressed in meristems and not in determinate lateral organs, to follow the branching events in ids1mum spikelets. knl is expressed in the spikel et and floral meristems of maize (Fig. 6A,B) (Jackson et al. 1994). Hybridization with a knl anti-sense probe on ids1mum2 spikelet meristems showed normal expression within the spikelet meristem (Fig. 6C). However, as soon as the ids1-mum2 spikelet meristem branches to form florets, the spikelet meristem becomes larger, and consequently, the domain of knl expression expands (Fig. $6 \mathrm{D})$. Strong knl expression is observed within the extra florets initiating from the spikelet meristem at later stages (Fig. 6E, arrowheads) confirming the meristematic origins of the extra florets. All other aspects of knl expression within the florets, including absence from the lateral organs and the LI layer, appear normal .

\section{Analysis of ids1 expression}

An initial characterization of idsl expression by use of RNA gel blots indicated that the gene was expressed in both vegetative and floral tissues. An RNA gel blot probed with the $3^{\prime}$ portion of the ids 1 CDNA shows that ids1 transcript is present in all tissues tested (Fig. 7A). The lowest amount of expression was seen in embryo tissue and the highest in inflorescence tissue.

To determine the levels of ids1 transcript in ids1mum mutants, poly $(A)^{+}$RN A was isolated from mutant ears and compared with RNA from wild-type ears. As shown in Figure 8A, no wild-type size transcript was found in either idsl-mum allele. Instead, faint, higher molecular weight transcripts were observed that may be the result of chimeric or alternatively spliced transcripts.
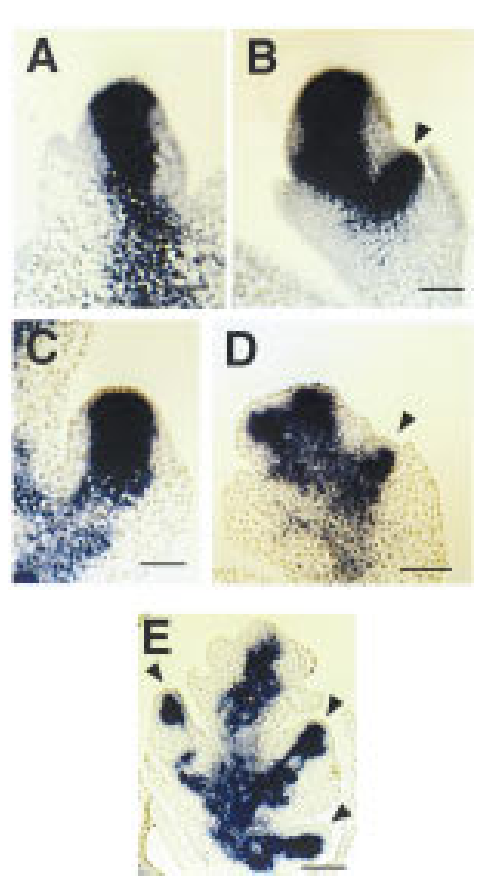

Figure 6. In situ localization of kn1 within A632 and ids1mum2 spikelet meristems. (A) kn1 expression within an unbranched A632 spikel et meristem. Bar, $50 \mu \mathrm{m}$. (B) kn1 expression within a branching A632 spikelet meristem. Expression can be seen in the initiating lower floret. Bar, $50 \mu \mathrm{m}$. (C) kn1 expression within an unbranched ids1-mum2 spikelet meristem. Bar, $50 \mu \mathrm{m}$. (D) kn1 expression within an early branching ids1-mum2 spikel et meristem. Initiating florets can be seen on both sides of the spikelet meristem. Bar, $60 \mu \mathrm{m}$. (E) kn1 expression within an older ids1-mum2 spikel et. Strong expression persists within the developing florets. Bar, $80 \mu \mathrm{m}$. A rrowheads point to initiating florets in $B, D$, and $E$. 
A

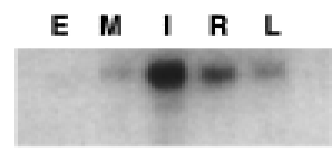

B

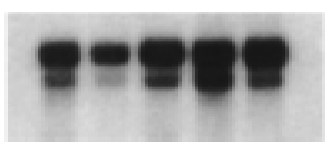

Figure 7. A nalysis of idsl expression in different organs of $B 73$ maize. (A) An RNA gel blot containing $1 \mu \mathrm{g}$ of poly(A) $)^{+}$RNA isolated from embryos 17 days postpollination (E), shoot meristems including unexpanded stem and the youngest leaf primordia (M), ear inflorescence primordia up to $2 \mathrm{~cm}$ long (I), primary roots from seeds germinated in humid air $(R)$, and the blade portion of fully expanded juvenile leaves $(L)$ was blotted and hybridized to the $3^{\prime}$ portion of the ids1 CDNA that does not include the AP2 domain. (B) Control hybridization. The blot in $A$ was probed with the maize ubiquitin CDNA to show relative loading.

This result demonstrates that the $\mathrm{Mu}$ element insertions into ids1 are not spliced out, and that both ids1-mum1 and ids1-mum2 may represent loss-of-function alleles.

To localize the temporal and spatial pattern of ids1 expression more precisely, we used in situ hybridization. Figure 9A shows longitudinal sections of a wild-type vegetative shoot apex probed with digoxigenin labeled antisense RN A made from the $3^{\prime}$ end of the idsl clone. The positions of leaves within the maize shoot have been described in terms of the plastochron index (Lamoreaux et al. 1978) in which the youngest leaf is referred to as plastochron 1 or $P_{1}$ and successive leaves as $P_{2}, P_{3}$, etc. The position of the incipient leaf in the meristem is referred to as $P_{0}$. ids 1 expression is detected on the flanks of the meristem in a discrete zone (Fig. 9A). From the position of the youngest leaf (label ed as $P_{1}$ ), we assume this zone of meristem expression is in the position in which the next leaf will initiate (labeled as $\mathrm{P}_{0}$ ). Expression is also seen in the tips of the next two ol der leaves, $\mathrm{P}_{1}$ and $\mathrm{P}_{2}$.

In Iongitudinal sections of floral tissue, idsl expression is detected in several different lateral organ primordia as well as the spikel et pair and spikelet meristems. In

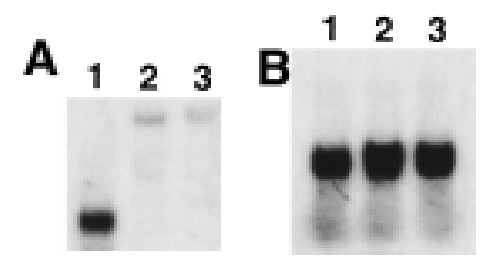

Figure 8. Analysis of idsl expression in ids1-mum mutant ears. (A) An RN A gel containing $1 \mu \mathrm{g}$ of poly(A) $)^{+} \mathrm{RN} A$ isolated from 2-cm ears from A 632 (1), ids1-mum2 (2), and ids1-mum1 (3) was blotted and hybridized to the $3^{\prime}$ portion of the ids1 CDN A. (B) Control hybridization. The bl ot in A was probed with the maize ubiquitin CDNA to show relative loading.

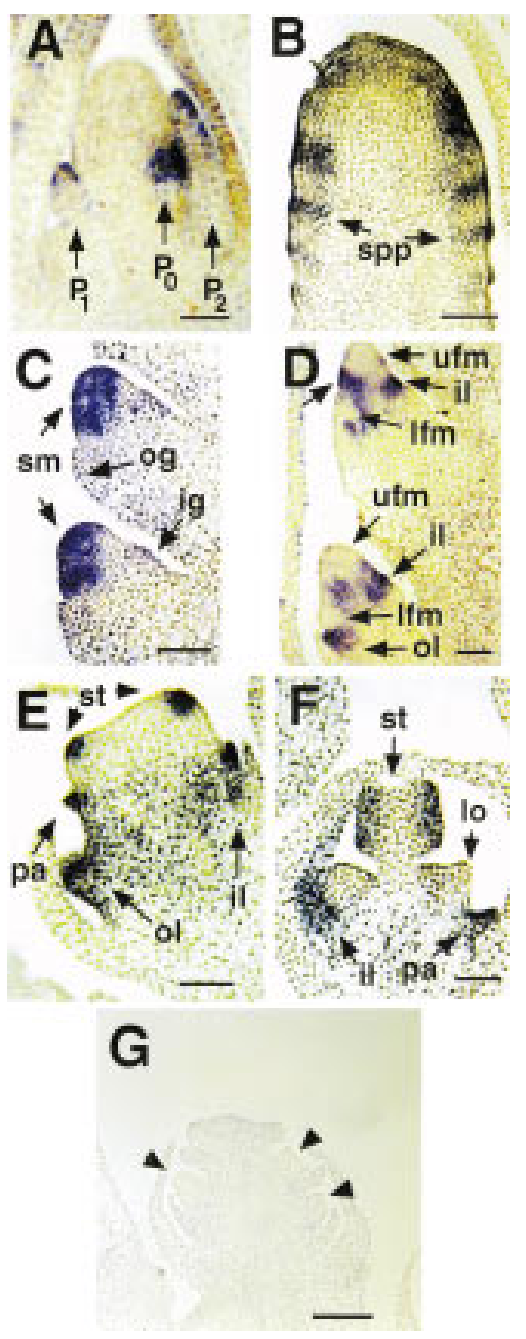

Figure 9. In situ localization in B73 vegetative and floral apices by use of the $3^{\prime}$ portion of the idsl CDNA. (A) Shoot apical meristem with young leaf primordia $\left(P_{1}\right.$ and $\left.P_{2}\right)$ and incipient leaf primordia $\left(P_{0}\right)$. Bar, $65 \mu \mathrm{m}$. (B) Y oung ear inflorescence. ids1 expression can be seen in the acropetally initiating spikel et pair primordia. Bar, $70 \mu \mathrm{m}$. (C) U nbranched spikelet meristems. Expression can be seen in the spikelet meristem but not in the glumes. Bar, $36 \mu \mathrm{m}$. (D) Branching spikel et meristems. Strong expression can be seen in the inner and outer lemmas as well as the zone in between the initiating upper and lower florets (unlabeled arrow). This zone of expression gradually disappears in the older spikelet below. No expression is detected in the initiating floral meristems. Bar, $70 \mu \mathrm{m}$. (E) Upper floret with initiating stamens. Discrete areas of idsl expression can be observed in the initiating stamen primordia and palea. ids1 expression persists in the expanding inner and outer lemmas. Bar, $70 \mu \mathrm{m}$. (F) Older floret with lodicules. Stamen expression now localizes to the locule chambers of the anther. No expression can be seen in the lodicules (Io). Bar, $70 \mu \mathrm{m}$. (G) ids1-mum2 spikelet probed with ids1. Little or no ids1 transcript can be detected. Arrowheads point to florets. Bar, $130 \mu \mathrm{m}$.

Figure 9B, longitudinal sections of early inflorescence meristems probed with ids1 show stripes of expression in a repeating pattern that evidently corresponds to spikel et pair primordia. At a later stage, after the pedi- 
cellate and sessile spikelets form, idsl expression is found throughout the spikelet meristem but not in the first two lateral organ primordia formed from the spikelet meristem, the outer and inner glumes (Fig. 9C). Soon after floral meristems initiate (Fig. 9D), idsl expression is present in the initiating inner and outer lemma bract leaves subtending the florets, but is absent from the floral meristem itself. In addition, a strong band of expression is seen in the region between the upper and lower florets (Fig. 9D, unlabeled arrow). In slightly older spikelets as shown at the bottom of Figure 9D, this band of expression begins to disappear. A scanning el ectron micrograph of spikelets at equivalent stages is shown in Figure 5B. In more mature tassel florets (Fig. 9E), discrete groups of cells of the floral meristem that are positioned to form the stamens and pal ea express idsl. Expression persists at later stages in the older expanded palea and lemma and also the locule chambers of the anther (Fig. 9F). Little or no expression was seen in the lodicules or the gynoecium of the ear (Fig. 9F; data not shown). As shown in Figure 9G, when an anti-sense idsl probe is used on idsl-mum spikelets, no idsl expression is detected.

\section{Discussion}

Regulating the number of florets initiated by the spikelet meristem is a critical step in the determination of spikelet morphology. Mutations within the idsl gene affect spikelet meristem determinacy. Instead of producing only an upper and lower floret, the ids1-mum spikelet meristem continues to initiate additional florets in a distichous phyllotaxy. Understanding how ids1 promotes spikel et meristem determinacy requi res an examination of different models for maize floret initiation.

One model for floret initiation suggests that the lower floret is initiated laterally whereas the upper floret is terminal, produced by the transformation of the spikelet meristem into the upper floret (Fig. 10A) (Irish 1997). In some grass species such as anthoxanthum, the spikelet terminates in a flower with the ovule being the terminal structure (Clifford 1987). If the upper floret is a terminal structure in normal maize development, the IDS1 protein may function to promote the transformation of the spikel et meristem into the upper floret. IDS1 may do this alone, or through the activation of floral meristem identity genes such as orthologs of LEAFY and APETELA 1 that can transform inflorescence meristems into floral meristems ( $M$ andel and Yanofsky 1995; Weigel and Nilsson 1995). If this were the case, we would speculate that the transformation of spikel et meristem to floral meristem is delayed in ids1-mum mutants, allowing the spikel et meristem to persist and initiate more florets. Such an interpretation has been invoked to explain the phenotype of the dominant Ts6 mutants of maize in which extra florets form (Irish 1997). Ts6 mutants differ from idsl-mum mutants, however, in that they also affect sex determination and cause an absence of carpel suppression within the tassel, but not in the ear.
A)
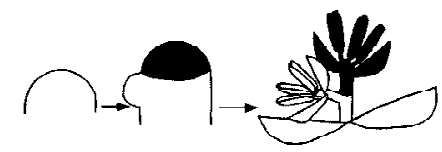

B)

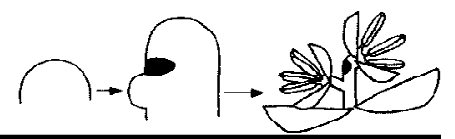

Figure 10. Models for maize floret initiation. (A) Terminal upper floret model. The spikel et meristem branches once laterally to form the lower floret. The resi dual spikel et meristem (shown in black) is then transformed into the upper floret (black). (B) Lateral branching model. The spikel et meristem undergoes lateral branching to form the lower and then upper florets. The residual spikel et meristem (shown in black) is found in a small region in between the florets. A rudiment of the spikelet meristem in found in the rachilla in the mature spikelet (black).

An alternative model would suggest that both upper and lower florets are lateral products of the spikel et meristem. The two fl orets of maize have been interpreted as lateral branches of the rachilla with little or no remnant of the axis left in between the florets (Bonnett 1953). If upper floret branching is lateral, then a greatly reduced rudiment of the spikel et meristem might be found in the rachilla after upper floret initiation (Fig. 10B). According to this model, IDS1 would participate in suppression of indeterminate growth within the spikelet meristem, thus preventing the spikel et meristem from regenerating after upper floret branching. In ids-mum mutants the residual spikel et meristem proliferates and continues to initiate florets.

On the basis of the expression pattern of idsl and the timing of the ids1-mum defects, we favor the second model and hypothesize that IDS1 functions to suppress indeterminate growth within the spikel et meristem. According to the first model, the switch in spikelet meristem fate occurs only after the lower floret is initiated. However, defects in ids1-mum spikelet meristems were first observed in the spikelet meristem prior to lower floret initiation (Fig. 5E). The timing of the defect suggests that the change in fate determination occurs earlier, in agreement with the idsl expression observed in the spikelet meristem before floret branching occurs (Fig. 9C). The first model also suggests that the entire spikelet meristem becomes transformed into an upper floret, leaving no residual spikel et meristem. Yet, ids1 expression was detected in a zone between the upper and lower florets in wild type (Fig. 9D) in which the residual spikel et meristem would be expected to be located. Additional evidence that this region represents the resi dual spikel et meristem comes from the observation that it regenerates after floret branching in ids1-mum mutants (Fig. 5F,G). Furthermore, an expectation of the first model is that ids1 would be expressed throughout the future upper floret to promote its transformation to floral identity. Instead, as shown in Figure 9D, we see no idsl expression in either the upper or lower floral meristems. Finally, if determinacy of the spikelet meristem 
were simply being delayed in idsl-mum mutants in accordance with the first model, one would expect to see the rachilla eventually terminate in a floret. This was not observed in either male and femal e ids1-mum spikelets (Fig. 4F; data not shown).

The development of a critical meristem size in maize is thought to provide the cue for branching to occur (Sundberg and Orr 1996). One mechanism by which IDS1 may suppress indeterminate growth is to restrict the size of the residual spikelet meristem such that it can no Ionger initiate florets. In ids1-mum mutants, the size of the residual spikel et meristem may be deregulated and expand to larger than normal. A larger spikel et meristem could potentially allow greater numbers of branching events to produce extra florets. In support of this idea, the ids1-mum spikelet meristem appears more elongated than normal before florets are initiated (Fig. 5E). Also, a larger spikelet meristem diameter is observed soon after the first floret is initiated in ids1-mum mutants (Fig. 6D).

It is important to recognize, however, that meristem fate is not always dependent on meristem size. For example, the upper floral meristem is much larger that the lower floral meristem at the time of initiation (Fig. 5B), and yet both are equally determined to make florets. Thus, having a larger spikel et meristem would not necessarily endow it with greater indeterminacy. An equally important component of the distinction between determinate and indeterminate spikel et meristem fates is the ability to regenerate after floret initiation. Meristems with indeterminate fates may show a greater ability at self-regeneration regardless of their initial size. The altered morphology seen in the spikelet meristems of the ids1-mum mutants may simply be a reflection of this ability.

It is possible that one way IDS1 functions to maintain spikel et meristem determi nacy is to repress those factors necessary for maintaining indeterminacy. The kn1 homeobox gene is expressed in several types of indeterminate meristematic tissues and not in determinate organs such as leaves (Smith et al. 1992; Jackson et al. 1994). Loss-of-function mutants of kn1 in maize show reduced tassel branching and fewer spikelets. This phenotype has been postulated to result from a loss of indeterminate inflorescence cells which, in the absence of knl, have adopted determinate fates (Kerstetter et al. 1997). If kn1 is in fact maintaining meristem indeterminacy, then kn1 expression should persist within the ids1-mum spikelet meristem and show an expanded domain. Although this was observed (Fig. 6D), it is important to note that $\mathrm{kn} 1$ is normally expressed in both spikelet and floral meristems (Fig. 6A,B). Thus, the fact that the domain of $\mathrm{knl}$ expression is expanded in idsl-mum mutants may simply reflect extra areas of floret initiation.

The idsl gene was cloned by homology to the AP2 gene of A rabidopsis and shown to contain two repeats of the highly conserved AP2 domain. Although the IDS1 protein shows greater similarity to AP2 compared with all other AP2-like genes cloned thus far, the idsl gene may not represent the actual AP2 orthol og of maize. The amino acid homology between IDS1 and AP2 does not extend outside of the AP2 domain. Furthermore, the expression of ids1 was not detected in carpels, as is found for AP2 (Jofuku et al. 1994). Finally, preliminary experiments show that the overexpression of the ids1 cDN A in Arabidopsis does not complement the ap2-1 mutant phenotype (G. Chuck unpubl.). Additional AP2 gene family members are likely to be present in the maize genome, because low stringency hybridizations with the ids1 AP2 domain as a probe on maize Southern blots show several hybridizing bands (data not shown). In addition, several expressed sequence tags from maize cDN A libraries have been shown to have AP2 domains (Klucher et al. 1996).

Even though idsl is expressed in vegetative and floral lateral organ primordia, no obvious mutant phenotypes were detected in those organs. A certain level of genetic redundancy may operate in these organs, which can only be uncovered through the analysis of double mutants. For example, double mutants of ap2 and aintegumenta show floral organ phenotypes not seen in either single mutant in Arabidopsis (Elliot et al. 1996). Considering the large number of AP2-like genes present in the ge nome of Arabidopsis (Okamuro et al . 1997), it would not be surprising to find such functional redundancy occurring in maize. Recently, it has been suggested that two closely related MADS box genes, zagl and zmm2, play redundant roles in specifying carpel and stamen identity in maize (M ena et al. 1996).

Although floral organs of ids1-mum tassel florets are fully functional and resemble those of wild type, floral organ defects were observed in idsl-mum female spikelets. A possible explanation for this defect may lie in the fact that the lower floret aborts in normal female spikelets. If the distinction between upper and lower florets is lost in ids1-mum mutants, then all the florets may receive the abortion signal that is normally only present in the lower floret in wild type. Double-mutant analysis with the tasseled 2 mutant, in which lower floret abortion does not occur (DeLong et al. 1993) will be useful to test this model.

In light of the fact that many members of the grass family possess indeterminate spikelets, it is tempting to speculate that AP2-like genes play a role in regulating floret number in other grasses. Spikelets with many florets are assumed to be an ancient trait given that derived species have almost universally evolved a reduction in floret number (Stebbins 1987). A possible mechanism for such a reduction may have involved a change in the function or domain of expression of the ids1 gene to include the spikelet meristem in addition to the various lateral organs where it is normal ly expressed. The analysis of idsl expression in both modern and ancient grasses will be interesting to analyze in this regard.

\section{Materials and methods}

Cloning the idsl gene

Forty thousand plaques of a B73 cDN A library constructed from 1- to 3-cm inflorescence ears (Jackson et al. 1994) were probed at 
reduced stringency at $55^{\circ} \mathrm{C}$ in $50 \%$ formamide (Schmidt et al. 1993) with the full-length AP2 CDN A from Arabidopsis (Jofuku et al. 1994). A pproximately 75 positive clones were isolated. Only the strongest hybridizing class was analyzed further. A representative clone from this class was used to probe a B73 vegetative meristem cDN A library (Lambda ZaplI, Stratagene) from which a full-length 1.9-kb cDN A was isolated. The CDN A was subcloned into pSK- and both strands sequenced with an $\mathrm{ABI}$ sequencer. Sequence analysis was done by use of GCG (Wisconsin Genetics Group) and the National Center for Biotechnology Information Blast E-MAIL server.

\section{RNA gel blots and in situ hybridization}

RNA was isolated from embryos, ears, vegetative meristems, and roots as described previously (Kerstetter et al. 1994). In situ hybridization was performed as described by Jackson et al. (1994) by use of a $3^{\prime}$ end probe between nucleotides 1330 and 1835 outside the conserved AP2 domain. This same DN A fragment was used to probe all RNA gel blots. A single band is observed to hybridize to this probe on DNA gel blots (data not shown), therefore, cross hybridization with other AP2 family members is unlikely.

\section{Isolation of recessive ids1 alleles by PCR}

Approximately $42,000 F_{1}$ plants from crosses between stocks containing active $\mathrm{Mu}$ transposable elements were grown and screened by PCR by Pioneer Hi-Bred International for insertion into ids1 (Bensen et al. 1995) with the ZAP2-1 primer (5'-CCGGTGGCGCCAGCGAAGAA-3') and Mu-9242 (5'-CCCTGAGCTCTTCGTC(CT)ATAATGGCAATTATCTC-3'), a degenerate primer that binds to the terminal inverted repeat of $\mathrm{Mu}$. PCR reactions were run on gels, blotted, and probed with the idsl CDN A. PCR products that hybridize to the idsl CDN A identified individuals that likely have $\mathrm{Mu}$ insertions into the idsl gene. Such a screen identified nine candi dates by use of the ZAP2-1 primer. DNA gel blot analysis of digested DNA made from self-pollinated progeny of each candidate revealed polymorphisms for only two of the nine families when probed with the idsl cDNA. These same two families were the only ones that generated ids1 PCR products by use of the ZAP2-1 and M u 9242 primers. It is likely that the seven other families that showed no polymorphisms represent somatic insertion events of $\mathrm{Mu}$ into ids1 that did not transmit into the germ line. Each ids1-mum allele was generated from a cross between a Mu active line and the A 632 inbred line. Both allel es were backcrossed to A632 once and self-pollinated to observe phenotypes in the homozygotes. The phenotypes were al so observed after a second backcross to $A 632$ and a backcross to inbred W23.

\section{Scanning el ectron microscopy}

Tissue was fixed in FAA (50\% ethanol, 5\% acetic acid, 3.7\% formal dehyde) at $4{ }^{\circ} \mathrm{C}$ overnight and dehydrated in a graded ethanol series to $100 \%$. The samples were then critical point dried and sputter coated with palladium. Samples were viewed on an ISI 30 SEM at $10 \mathrm{kV}$ accelerating voltage.

\section{Acknowledgments}

We thank J. Okamuro and D. Jofuku (U niversity of California, Santa Cruz) for the gift of the AP2 CDNA, R. Kerstetter for the use of his RNA blot, and K. Canada (Pioneer Hi-Bred International) for identifying idsl insertions. We are al so grateful to $\mathrm{E}$. Vollbrecht, P. M cSteen, L. Reiser, F. Hempel, and the members of the Hake laboratory for reviewing the manuscript and providing hel pful discussions. G.C. was supported by a U niversity of California fellowship. This work was supported by the U.S. Department of Agriculture and performed, in part, under a Cooperative Research and Development Agreement with Pioneer $\mathrm{Hi}$-Bred International, Inc.

The publication costs of this article were defrayed in part by payment of page charges. This article must therefore be hereby marked "advertisement" in accordance with 18 USC section 1734 solely to indicate this fact.

\section{References}

Bennetzen, J.L., P.S. Springer, A.D. Cresse, and M. Hendrickx. 1993. Specificity and regulation of the Mutator transposable element system in maize. Critical Rev. Plant Sci. 12: 57-95.

Bensen, R.J., G.S. Johal, V.C. Crane, J.T. Tossberg, and P.S. Schnable. 1995. Cloning and characterization of the maize An1 gene. Plant Cell 7: 75-84.

Bonnett, O.T. 1953. Developmental morphology of the vegetative and floral shoots of maize. III. Agric. Exp. Stn. Bull. 568.

Bossinger, G., W. Rohde, U. Lundqvist, and F. Salamini. 1992. Genetics of barley development: Mutant phenotypes and molecular aspects, In Barley: Genetics, biochemistry, molecular biology and biotechnology, (ed. P.R. Shewry), pp. 231-264. Alden Press Ltd, Oxford, UK.

Bowman, J.L., J. Alvarez, D. Weigel, E.M. Meyerowitz, and D.R. Smyth. 1993. Control of flower development in A rabidopsis thaliana by APETALA 1 and interacting genes. Devel opment 119: 721-743.

Burr, B., F.A. Burr, K.H. Thompson, M.C. Albertson, and C.W. Stuber. 1988. Gene mapping with recombinant inbreds in maize. Genetics 118: 519-526.

Cheng, P.C., R.I. Greyson, and D.B. Walden. 1983. Organ initiation and the development of unisexual flowers in the tassel and ear of Zea mays. Am. J. Bot. 70: 450-462.

Clifford, H.T. 1987. Spikelet and floral morphology, in Grass systematics and evolution, (ed. T.R. Soderstrom, K.W. Hilu, C.S. Campbell, and M.E. Barkworth), pp. 21-30. Smithsonian Institution Press, Washington, DC.

Clifford, H.T. and L. Watson. 1977. Identifying grasses: Data, methods and illustrations. University of Queensland Press, St. Lucia, Queensland, Australia.

Dellaporta, S.L. and A. Calderon-U rrea. 1993. Sex determination in flowering plants. Plant Cell 5: 1241-1251.

Delong, A., A. Calderon-Urrea, and S.L. Dellaporta. 1993. Sex determination gene TASSELSEED2 of maize encodes a short-chain alcohol dehydrogenase required for stage-specific floral organ abortion. Cell 74: 757-768.

Elliot, R.C., A.S. Betzner, E. Huttner, M.P. Oakes, and W.Q.J. Tucker. 1996. AINTEGU MENTA, an APETALA2-like gene of Arabidopsis with pleiotropic roles in ovule development and floral organ growth. Plant Cell 8: 155-168.

Hempel, F.D. and L.J. Feldman. 1994. Bi-directional inflorescence development in Arabidopsis thaliana: Acropetal initiation of flowers and basipetal initation of paraclades. Planta 192: 276-286.

Irish, E.E. 1997. Experimental analysis of tassel development in the maize mutant Tassel seed 6. Plant Physiol. 114: 817-825.

Irish, E.E., J.A. Langdale, and T.M. N elson. 1994. Interactions between tassel seed genes and other sex determining genes in maize. Dev. Genetics 15: 155-171.

Jackson, D., B. Veit, and S. Hake. 1994. Expression of maize KNOTTED 1 related homeobox genes in the shoot apical meristem predicts patterns of morphogenesis in the vegetative shoot. Development 120: 405-413. 
Jofuku, K.D., B.G.W. den Boer, M. Van Montagu, and J.K. Okamuro. 1994. Control of Arabidopsis flower and seed development by the homeotic gene APETALA2. Plant Cell 6: 1211-1225.

Kempton, J.H. 1934. Heritable characters in maize. XLVII Branched silkless. J. Hered. 25: 29-32.

Kerstetter, R., E. Vol Ibrecht, B. Lowe, B. Veit, and J. Yamaguchi. 1994. Sequence analysis and expression patterns divide the maize knotted1-like homeobox genes into two classes. Plant Cell 6: 1877-1887.

Kerstetter, R.A., D. Laudencia-Chingcuanco, L.G. Smith, and S. Hake. 1997. Loss of function mutations in the maize homeobox gene, knotted 1 , are defective in shoot meristem maintenance. Development 124: 3045-3054.

Klucher, K.M., H. Chow, L. Reiser, and R.L. Fischer. 1996. The AINTEGU MENTA gene of Arabidopsis required for ovule and female gametophyte devel opment is related to the floral homeotic gene APETALA2. Plant Cell 8: 137-153.

Kunst, L., J.E. Klenz, J. Martinez-Zapater, and G.W. Haughn. 1989. The AP2 gene determines the identity of perianth organs in flowers of Arabidopsis thaliana. Plant Cell 1: 11951208.

Lamoreaux, R.J., W.R. Chaney, and K.M. Brown. 1978. The plastochron index: A review after two decades of use. Am. J. Bot. 65: 586-593.

M andel, M.A. and M .F. Yanofsky. 1995. A genetriggering flower formation in Arabidopsis. Nature 377: 522-524.

Meeley, R.B. and S.P. Briggs. 1995. Reverse genetics for maize. Maize Genet. Coop. Newsl. 69: 67,82.

Mena, M., B. Ambrose, R.B. Meeley, S.P. Briggs, and M.F. Yanofsky. 1996. Diversification of C-function activity in maize flower devel opment. Science 274: 1537-1540.

Modrusan, Z., L. Reiser, K.A. Feldmann, R.L. Fischer, and G.W. Haughn. 1994. Homeotic transformation of ovules into carpel-like structures in Arabidopsis. Plant Cell 6: 333-349.

Moose, S. and P. Sisco. 1997. Glossy15, an APETALA2-like gene from maize that regulates leaf epidermal cell identity. Genes \& Dev. 10: 3018-3027.

Ohme-Takagi, M. and H. Shinshi. 1995. Ethylene-inducible DNA binding proteins that interact with an ethylene-re sponsive element. Plant Cell 7: 173-182.

Okamuro, J.K., B. Caster, R. Villarroel, M. Van Montagu, and D.K. Jofuku. 1997. The AP2 domain of APETALA 2 defines a large new family of DNA binding proteins in Arabidopsis. Proc. Natl. Acad. Sci. 94: 7076-7081.

Ougham, H.J., G. Latipova, and J. Valentine. 1996. M orphological and biochemical characterization of spikelet development in naked oats (Avena Sativa). New Phytol. 134: 5-12.

Randolph, L.F. 1936. Developmental morphology of the caryopsis in maize. J. Agric. Res. 53: 881-916.

Schmidt, R.J., B. Veit, M.A. Mandel, M. Mena, and S. Hake. 1993. Identification and molecular characterization of ZAG 1 the maize homolog of the Arabidopsis floral homeotic gene AGAMOUS. Plant Cell 5: 729-737.

Schultz, E.A. and G.W. Haughn. 1993. Genetic analysis of the floral initiation process (FLIP) in Arabidopsis. Development 119: 745-765.

Smith, L.G., B. Greene, B. Veit, and S. Hake. 1992. A dominant mutation in the maize homeobox gene, Knotted 1 causes its ectopic expression in leaf cells with altered fates. Development 116: 21-30.

Stebbins, G.L. 1987. Grass systematics and evolution: Past, present and future, In Grass systematics and evolution, (ed. T.R. Soderstrom, K.W. Hilu, C.S. Campbell, and M.E. Barkworth), pp. 359-367. Smithsonian Institution Press, Washington, D.C.
Sundberg, M.D. and A.R. Orr. 1996. Early inflorescence and floral development in Zea mays land race chapal ote (Poaceae). Am. J. Bot. 83: 1255-1265.

Veit, B., R.J. Schmidt, S. Hake, and M.F. Yanofsky. 1993. Maize floral development-new genes and old mutants. Plant Cell 5: 1205-1215.

Weatherwax, P. 1923. The story of the maize plant. The University of Chicago Press, Chicago, IL.

Weigel, D. and O. Nilsson. 1995. A developmental switch sufficient for flower initiation in diverse plants. Nature 377: 495-500. 


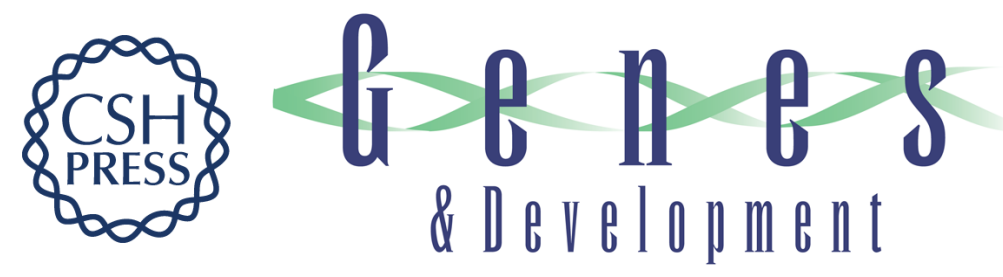

\section{The control of maize spikelet meristem fate by theAPETALA2-like gene indeterminate spikelet1}

George Chuck, Robert B. Meeley and Sarah Hake

Genes Dev. 1998, 12:

References This article cites 32 articles, 21 of which can be accessed free at:

http://genesdev.cshlp.org/content/12/8/1145.full.html\#ref-list-1

License

Email Alerting
Service

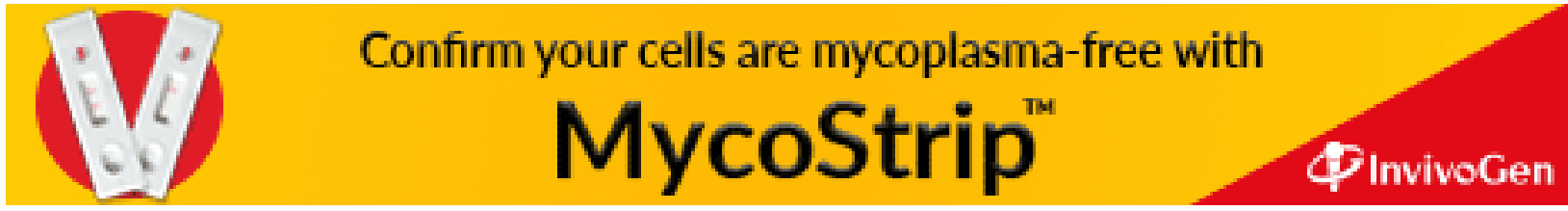

